\title{
A guide to managing disorders of the ear pinna and canal
}

\section{This review will help you troubleshoot everything from infections and foreign bodies to trauma and neoplasm.}

\section{PRACTICE RECOMMENDATIONS \\ > Prescribe topical antibiotics for uncomplicated otitis externa, reserving systemic agents for infection extending outside the ear canal, necrotizing otitis externa, or patients who are immunodeficient. (C)}

> Avoid clearing cerumen if a patient is asymptomatic and advise patients/parents on Do's and Don'ts for ear wax accumulation. (C)

> Consider flooding the ear canal with xylocaine, alcohol, or mineral oil before attempting insect removal. (C)

Strength of recommendation (SOR)

A Good-quality patient-oriented evidence

B Inconsistent or limited-quality patient-oriented evidence

C Consensus, usual practice, opinion, disease-oriented evidence, case series
$\mathrm{W}$ hich antibiotics are most useful for infection following ear piercing? When is it safe to attempt removal of a foreign body from the ear canal, and which cerumenolytic agent may be best for ear wax? This review covers common ailments of the outer ear, which are often readily diagnosed given a patient's history and thorough physical examination. We also address more complicated matters such as deciding when to refer for treatment of suspected malignant otitis externa, and which lab markers to follow when managing it yourself.

\section{A (very) brief review of ear anatomy}

Understanding the unique embryology and intricate anatomy of the external ear informs our understanding of predictable infections, growths, and malformations.

The external ear is composed of the external auditory canal and auricle. The external auditory canal has a lateral (external) cartilaginous portion and a medial (internal) bony portion. The auricular structure is complex and formed by the helix, antihelix (crura; scaphoid fossa), tragus, antitragus, conchae, and lobule. The auricle is composed of elastic cartilage covered by skin. The lobule is composed of skin, adipose tissue, and connective tissue.

Embryologically, the auricle, auditory canal, and middle ear form from ectoderm of the first 2 branchial arches during early gestation. The auricle forms from the fusion of soft-tissue swellings (hillocks). Three hillocks arise from the first branchial arch and 3 from the second branchial arch during the fifth and sixth weeks of gestation. Tissues from the second branchial arch comprise the lobule, antihelix, and caudal helix. The cartilage of the tragus forms from the first branchial arch. The ear canal forms from an epithelial invagination of the first branchial arch that also occurs during the fifth week of gestation. ${ }^{1}$

\section{Infections}

Perichondritis

Inflammation or infection of the connective tissue layer sur-
Kristen Grine, DO; Mark Stephens, MD Department of Family and Community Medicine, Penn State College of Medicine, University Park

- $m$ mstephens3@ pennstatehealth.psu.edu

The authors reported no potential conflict of interest relevant to this article. 


\section{$>$}

\section{Rates of} infection as high as $35 \%$ have been reported with high-ear piercing. rounding the auricular cartilage (perichondrium) results in perichondritis. Further extension of infection can lead to an auricular abscess. Both of these conditions can have serious consequences.

I What you'll see. The most common risk factor for perichondritis is the popular practice of cosmetic transcartilaginous piercing. ${ }^{2}$ Piercing of the helix, scapha, or antihelix (often referred to as "high" ear piercing) causes localized trauma that can strip the adjacent perichondrium, decrease blood supply, create cartilaginous microfractures, and lead to devascularization. Rates of infection as high as $35 \%$ have been reported with highear piercing. ${ }^{3}$

The most common microbes associated with perichondritis and pinna abscess formation are Pseudomonas and Staphylococcus species. ${ }^{2}$ P aeruginosa accounts for a majority $(87 \%)$ of post-piercing infections of the auricular cartilage. ${ }^{2}$

I How to treat. The cornerstone of treatment is early detection and antimicrobial coverage with antipseudomonal antibiotics. Ciprofloxacin is the oral antibiotic of choice because of its ability to penetrate the tissue. ${ }^{4}$ Other options include clindamycin and third- or fourth-generation cephalosporins. If the wound becomes abscessed, perform (or refer for) early surgical incision and drainage. $^{5} \mathrm{~A}$ failure to promptly recognize perichondritis or to mistakenly prescribe nonantipseudomonal antibiotics contributes to increased rates of hospitalization. ${ }^{2}$ Cosmetic deformity is the most common complication of perichondritis. This may require reconstructive surgery.

\section{Otitis externa}

Acute otitis externa (AOE; "swimmer's ear") is cellulitis of the skin and subdermis of the external ear canal. It is most prevalent in warm, moist climates and almost always associated with acute bacterial infection, most commonly P aeruginosa or $S$ aureus. ${ }^{6}$ There is also an increased association with poor water quality (containing higher bacterial loads). Anything breaching the integrity of the ear canal can potentially predispose to the development of AOE. This includes trauma from cleaning, cerumen removal, scratching due to allergic conditions, and placement of hearing-aid devices. ${ }^{6}$

I What you'll see. Suspect AOE when signs or symptoms of ear canal inflammation have appeared rapidly (generally within 2 days) over the past 3 weeks. ${ }^{7}$ Findings include otalgia, itching, fullness, tragal tenderness, ear canal edema, erythema with or without otorrhea, lymphadenitis, or cellulitis of the pinna or adjacent skin. ${ }^{7}$ AOE must be distinguished from other causes of otalgia and otorrhea, including dermatitis and viral infection.

I How to treat. Topical therapy is recommended for the initial treatment of uncomplicated AOE, usually given over 7 days. Multiple topical preparations are available, such as ciprofloxacin $0.2 \%$ /hydrocortisone $1.0 \%$; neomycin/polymyxin B/hydrocortisone; ofloxacin $0.3 \%$; or acetic acid $2.0 \%{ }^{7}$ Avoid these agents, though, if you suspect tympanic membrane rupture. Quinolone drops are the only topical antimicrobials approved for middle ear use. ${ }^{7}$

Systemic antibiotics are not recommended for the initial treatment of AOE. Topical agents deliver a much higher concentration of medication than can be achieved systemically. Consider systemic antibiotics if there is extension outside the ear canal, a concern for necrotizing otitis externa (more on this in a bit), or the patient is immunodeficient. ${ }^{8}$

Patient (or parent) education is important to ensure proper medication administration. The patient should lie down with the affected ear facing up. After the canal is filled with drops, the patient should remain in this position for 3 to 5 minutes. Gently massaging the tragus can augment delivery. Patients should keep the ear canal as dry as possible and avoid inserting objects (eg, hearing aids, ear buds, cotton-tipped applicators) into the canal for the duration of treatment. The delivery of topical antibiotics can be enhanced by wick placement. Prescribe analgesics (typically nonsteroidal anti-inflammatory agents) based on severity of pain. ${ }^{7}$

Have patients abstain from water sports for 7 to 10 days. Showering is acceptable with minimal ear exposure to water; bathing is preferred when possible. If there is no clinical improvement in 48 to 72 hours, ask 
patients to return for re-evaluation. ${ }^{8}$ Prevention is essential for patients with a history of recurrent otitis externa. Acetic acid solutions create an acidic environment within the canal to help prevent recurrent AOE. Ear plugs and petroleum jelly-soaked cotton plugs prior to water exposure may also help prevent recurrent AOE.

\section{Malignant otitis externa}

Malignant, or necrotizing, otitis externa is an aggressive disease form of otitis externa that is most common in individuals with diabetes or other immunodeficiency disorders. ${ }^{9}$ Most cases are due to infection with $P$ aeruginosa.$^{10}$ Prior to the availability of effective antibiotics, mortality rates in patients with necrotizing otitis externa were as high as $50 \% .{ }^{11}$

I What you'll see. Patients typically present with severe ear pain, otorrhea, conductive hearing loss, and a feeling of fullness in the external ear canal. Physical examination reveals purulent otorrhea and a swollen, tender ear canal. Exposed bone may be visible, most often on the floor of the canal. The tympanic membrane and middle ear are seldom involved on initial presentation.

The infection often originates at the junction of the bony and cartilaginous portion of the external canal, spreading through the fissures of Santorini to the skull base. If not aggressively treated, the infection spreads medially to the tympanomastoid suture causing intracranial complications-usually a facial nerve neuropathy.

Given these clinical findings, promptly order laboratory studies and imaging to confirm the diagnosis. The erythrocyte sedimentation rate and C-reactive protein level are typically elevated, and either can be used as a marker to follow treatment. Computed tomography (CT) helps to determine the location and extent of disease and is recommended as the initial diagnostic imaging modality for patients with suspected malignant otitis externa. ${ }^{12}$

Magnetic resonance imaging helps define soft-tissue changes, dural enhancement, and involvement of medullary bone, making this the preferred modality to monitor therapeutic response. ${ }^{12}$ Technetium bone scanning can also be used for the initial diagnosis (particularly if CT findings are normal and clinical suspicion is high) and for follow-up with treatment.

I How to treat. Management involves a team approach with otolaryngology, radiology, neurology, endocrinology, and infectious disease specialists. Long term (6-8 weeks) antipseudomonal antibiotic treatment is typical.

Let culture results guide the choice of antibiotic. Fluoroquinolone therapy, usually ciprofloxacin, is used most often. ${ }^{12}$ Surgical intervention may be required for local debridement and drainage of abscesses. Close follow-up is necessary due to reports of recurrence up to 1 year after treatment. If left untreated, necrotizing otitis externa can lead to osteomyelitis, meningitis, septic thrombosis, cerebral abscess, and death. ${ }^{11}$

\section{Cerumen impaction}

The relatively small diameter of the external auditory canal increases the risk for impaction of cerumen and foreign bodies. Cerumen impaction, in particular, is a common primary care complaint. Cerumen forms when glandular secretions from the outer two-thirds of the ear canal mix with exfoliated skin. It functions as a lubricant for the ear canal and as a barrier against infection, water accumulation, and foreign bodies. ${ }^{13}$

I What you'll see. You may encounter cerumen impaction in an asymptomatic patient when it prevents visualization of the external auditory canal or tympanic membrane, or when a patient complains of conductive hearing loss, tinnitus, dizziness, ear pain, itching, and cough. ${ }^{13}$ It is found in 1 in 10 children and 1 in 20 adults. ${ }^{13}$ There is a higher incidence in patients who are elderly, are cognitively impaired, or wear hearing devices or ear plugs. ${ }^{13,14}$ Asymptomatic cerumen impaction should not be treated. A recent clinical guideline provides a useful "do and don' $t$ " list for patient education (TABLE). ${ }^{13}$

I How to treat. In asymptomatic patients, the presence of cerumen on examination is not an indication for removal. Based on current guidelines, ${ }^{13}$ impacted cerumen can safely be removed from the ear canal of symptomatic patients in several ways:
In head-tohead laboratory comparisons, distilled water appears to be the best cerumenolytic. 
TABLE

Do's and don'ts for patients ${ }^{13}$

\begin{tabular}{l|l}
\hline Do & Don't \\
\hline Leave earwax alone if not causing symptoms & Excessively clean your ear canal \\
\hline $\begin{array}{l}\text { Remove ear wax if it ... } \\
\text { - affects your hearing }\end{array}$ & Place small objects in the ear, including cotton tips. \\
- causes ringing in your ear & \\
- causes irritation & \\
- causes hearing device malfunction & \\
\hline $\begin{array}{l}\text { See your health care provider if you experience } \\
\text { ear pain, drainage, or bleeding }\end{array}$ & Use ear candles \\
\hline Keep hearing devices clean & Use drops if eardrum could be/is ruptured \\
\hline
\end{tabular}

- Manual removal with cerumen loop/ spoon or alligator forceps. This method decreases the risk for infection because it limits moisture exposure. However, it should be performed by a health care provider trained in its use because of the risk for trauma to the ear canal and tympanic membrane.

- Irrigation of the ear using tap water or a 50-50 solution of hydrogen peroxide and water. Irrigation can be achieved with a syringe or jet irrigator using a modified tip. This method also has a risk for trauma to the ear canal and tympanic membrane and should only be performed by appropriately trained health care professionals.

- Use of cerumenolytic agents to soften and thin earwax and promote natural extrusion. Several types of cerumenolytic drops (water-based and oil-based) are available and appear to be equally effective. Water-based solutions contain hydrogen peroxide, docusate sodium, acetic acid, and sodium bicarbonate. Oil-based drops may contain peanut, almond, or olive oils. A thorough allergic history should be performed to avoid using products in patients with nut allergies. In headto-head laboratory comparisons, distilled water appears to be the best cerumenolytic. ${ }^{15}$

\section{Foreign bodies}

Foreign bodies in the external auditory canal (typically beads, cotton tips, and insects) are more common in children than adults. ${ }^{16}$

I What you'll see. Most foreign bodies are lodged in the bony part of the external auditory canal, and many patients try to remove the object before seeking medical care. Removal requires adequate visualization and skill. ${ }^{17}$ Although patients may be asymptomatic, most complain of pain, fullness, decreased hearing, or otorrhea.

I How to treat. Directly visible objects can often be removed without referral. Suction, irrigation, forceps, probes, and fine hooks have been used. Insect removal can be facilitated by first flooding the canal with xylocaine, alcohol, or mineral oil. Acetone may be used to dissolve foreign bodies containing Styrofoam or to loosen glues. If the object is a button battery, avoid irrigation to prevent liquefaction tissue necrosis.

Complications of foreign body removal include pain, otitis externa, otitis media, and trauma to the ear or tympanic membrane. The likelihood of successful removal of the object decreases and the risk for complications increases with each subsequent attempt. ${ }^{17}$ Consult an otolaryngologist if sedation or anesthesia is required, the foreign body is tightly wedged, there is trauma to the ear canal or tympanic membrane, the foreign body has a sharp edge (eg, glass or wire), or removal attempts have been unsuccessful.

\section{Trauma}

Sports injuries, motor vehicle accidents, bites, falls, and burns are the primary causes of trauma to the external ear. ${ }^{18}$ 
I What you'll see. Blunt auricular trauma predisposes to infection, necrosis, and scar contracture. One of the most common sequelae is cauliflower ear. Trauma is particularly common with contact sports such as boxing, wrestling, or mixed martial arts. The skin of the auricle attaches directly to the perichondrium. Following blunt or shearing trauma to the auricle, hematomas form within the space between the perichondrium and cartilage of the anterior ear. ${ }^{19}$ Following hematoma formation, the healing process brings chondrocytes and fibroblasts to the subperichondrial space, promoting fibrocartilage formation. Over time (and with repeated injury), this can lead to a chronic, bulbous deformity known as cauliflower ear.

I How to treat. Small hematomas can be managed by aspiration, while larger ones generally require open drainage..$^{20}$ Newer treatments involving pressure dressings and the use of fibrin glue have been proposed. ${ }^{20}$ Recommend that athletes participating in contact sports wear appropriate protective headgear to prevent auricular hematoma and cauliflower ear.

\section{Neoplasm}

Roughly $5 \%$ of all skin cancers involve the ear, most frequently the pinna due to chronic sun exposure. ${ }^{21}$ The most frequently occurring malignancy of the external ear is basal cell carcinoma (BCC), which is responsible for $80 \%$ of all nonmelanoma skin cancers. ${ }^{22}$

I What you'll see. BCC of the ear usually involves the preauricular area and the helix. The risk for BCC is related to exposure to ultraviolet radiation. BCC of the ear is more common in men and can be particularly aggressive, highlighting the importance of prevention and prompt recognition. BCC typically presents as a fleshy papule that is often translucent or "pearly" and has overlying telangiectasia and a "rolled" border. Central ulceration can occur as well.

I How to treat. Usual treatment of BCC is surgical excision. Prevention is critical and centers on sun avoidance or the use of appropriate sunscreens.

In addition to BCC, exposure of the external ear to sunlight and ultraviolet ra- diation predisposes patients to the development of squamous cell carcinoma (SCC) and melanoma. SCC has a variety of presentations including papules, plaques, and nodules. SCC has a higher metastatic potential than does BCC.

\section{Keloid}

Keloids are an abnormal healing response to soft-tissue injury: benign fibrocartilaginous growths that extend beyond the original wound.

I What you'll see. Keloids are more common in dark-skinned individuals and tend to result from burns, surgical incisions, infection, trauma, tattooing, injections, piercings, and arthropod bites. In some cases, they arise spontaneously. Keloids are more common in areas of increased skin tension (chest, shoulders, back), but may occur on the ears-most commonly after piercing or trauma. Keloids present clinically as slow-growing rubbery or firm nodules. The diagnosis is typically based on clinical appearance but can be confirmed by histopathology.

I How to treat. Treatments vary and include observation, excision, intralesional injections, cryotherapy, enzyme therapy, silicone gel application, and irradiation..$^{23}$ Recurrence is common; no therapy has been proven to be universally superior or preferred.

\section{Congenital malformations Atresia}

Disruption of embryologic development (failed invagination of the external auditory canal) can lead to a stenotic or absent ear canal (aural atresia). Aural atresia is also often associated with fusion of the incus and malleus. This condition occurs predominantly in males. Unilateral atresia is more common than bilateral atresia, and the right ear is more often involved than the left. ${ }^{24}$

\section{Microtia}

Microtia is the incomplete development of the pinna leading to a small or deformed pinna. Microtia can be unilateral or bilateral. As with atresia, microtia more commonly af-
If the object in the external auditory canal is a button battery, avoid irrigation to prevent liquefaction tissue necrosis. 


\section{$>$}

Directly visible foreign objects can often be removed without referral, but the likelihood of success decreases with each subsequent attempt. fects males and, if unilateral, the right side is more often affected than the left. Microtia can occur in isolation but is often associated with genetic syndromes such as Treacher Collins syndrome and craniofacial microsomia (Goldenhar syndrome). When microtia is identified (typically at birth or early infancy), audiologic testing and a thorough physical examination for evidence of associated defects should be performed. Consult with an audiologist, clinical geneticist, or pediatric otolaryngologist.

\section{Pre-auricular pits}

Pre-auricular pits (sinuses) are tiny indentations anterior to the helix and superior to the tragus. While pre-auricular pits are more common on the right side, they are bilateral in $25 \%$ to $50 \%$ of cases. ${ }^{25}$ Pre-auricular pits occur in up to $1 \%$ of white children, $5 \%$ of black children, and $10 \%$ of Asian children. ${ }^{25}$ Children with this condition should undergo formal audiologic testing as their risk for hearing loss is higher compared with the general population. ${ }^{26}$

The branchio-oto-renal syndrome (associated with pre-auricular pits and hearing loss) also features structural defects of the ear, renal anomalies and/or nasolacrimal duct stenosis or fistulas. If this syndrome is suspected, renal ultrasound imaging is warranted. Other indications for renal ultrasound in patients with a pre-auricular pit are any dysmorphic feature, a family history of deafness, an auricular malformation, or a maternal history of gestational diabetes. ${ }^{27}$ Pre-auricular pits do not require surgery unless they drain chronically or become recurrently infected. Complete surgical excision is the treatment of choice in these cases.

JFP

\section{CORRESPONDENCE}

Mark Stephens, MD, 1850 Park Avenue, State College, PA 16801; mstephens3@pennstatehealth.psu.edu

\section{References}

1. Cox TC, Camci ED, Vora S, et al. The genetics of auricular development and malformation: new findings in model systems driv- ing future directions for microtia research. Eur J Med Genet. 2014;57:394-401.

2. Sosin M, Weissler JM, Pulcrano M, et al. Transcartilaginous ear piercing and infectious complications: a systematic review and critical analysis of outcomes. Laryngoscope. 2015;125:18271834 .

3. Stirn A. Body piercing: medical consequences and psychological motivations. Lancet. 2003;361:1205-1215.

4. Liu ZW, Chokkalingam P. Piercing associated perichondritis of the pinna: are we treating it correctly? J Larygol Oncol. 2013;127:505-508.

5. Mitchell S, Ditta K, Minhas S, et al. Pinna abscesses: can we manage them better? A case series and review of the literature. Eur Arch Otorhinolaryngol. 2015;272:3163-3167.

6. Stone KE. Otitis externa. Pediatr Rev. 2007;28:77-78.

7. Rosenfeld RM, Schwartz SR, Cannon CR, et al. Clinical practice guideline: acute otitis externa. Otolaryngol Head Neck Surg. 2014;150(1 suppl):S1-S24.

8. Prentice P. American Academy of Otolaryngology: Head and Neck Surgery Foundation clinical practice guideline on acute otitis externa. Arch Dis Child Educ Pract Ed. 2015;100:197.

9. Unadkat S, Kanzara T, Watters G. Necrotising otitis externa in the immunocompetent patient. J Laryngol Otol. 2018;132:71-74.

10. Carfrae MJ, Kesser BW. Malignant otitis externa. Otolarngol Clin NAm. 2008;41:537-549.

11. Chandler JR, Malignant otitis externa. Laryngoscope. 1968;78:1257-1294.

12. Hollis S, Evans K. Management of malignant (necrotising) otitis externa. J Laryngol Otol. 2011;125:1212-1217.

13. Schwartz SR, Magit AE, Rosenfeld RM, et al. Clinical practice guideline (update): earwax (cerumen impaction). Otolaryngol Head Neck Surg. 2017;156:S1-S29.

14. Guest JF, Greener MJ, Robinson AC, et al. Impacted cerumen: composition, production, epidemiology and management. QJM. 2004;97:477-488.

15. Saxby C, Williams R, Hickey S. Finding the most effective cerumenolytic. J Laryngol Otol. 2013;127:1067-1070.

16. Awad AH, ElTaher M. ENT foreign bodies: an experience. Int Arch Otorhinolaryngol. 2018;22:146-151.

17. Heim SW, Maughan KL. Foreign bodies in the ear, nose, and throat. Am Fam Physician. 2007;76:1185-1189.

18. Sharma K, Goswami SC, Baruah DK. Auricular trauma and its management. Indian $J$ Otolaryngol Head Neck Surg. 2006;58:232-234.

19. Haik J, Givol O, Kornhaber R, et al. Cauliflower ear-a minimally invasive treatment in a wrestling athlete: a case report. Int Med Case Rep J. 2018;11:5-7.

20. Ebrahimi A, Kazemi A, Rasouli HR, et al. Reconstructive surgery of auricular defects: an overview. Trauma Mon. 2015;20: e28202.

21. Warner E, Weston C, Barclay-Klingle N, et al. The swollen pinna. BMJ. 2017; 359; j5073.

22. Rubin AI, Chen EH, Ratner D. Basal cell carcinoma. $N$ Engl J Med. 2005;353:2262-2269.

23. Ranjan SK, Ahmed A, Harsh V, et al. Giant bilateral keloids of the ear lobule: case report and brief review of the literature. J Family Med Prim Care. 2017;6:677-679.

24. Roland PS, Marple BF. Disorders of the external auditory canal. J Am Acad Audiol. 1997;8:367-378.

25. Scheinfeld NS, Silverberg NB, Weinberg JM, et al. The preauricular sinus: a review of its clinical presentation, treatment, and associations. Pediatr Dermatol. 2004;21:191-196.

26. Roth DA, Hildesheimer M, Bardestein S, et al. Preauricular skin tags and ear pits are associated with permanent hearing impairment in newborns. Pediatrics. 2008;122:e884-890.

27. Tan T, Constantinides H, Mitchell TE. The preauricular sinus: a review of its aetiology, clinical presentation and management. Int J Ped Otorhinolaryngol. 2005;69:1469-1474. 